\title{
Télescope
}

Revue d'analyse comparée en administration publique

\section{De la liberté du logiciel et de son ouverture : tour d'horizon et perspectives}

\section{Guillaume Blum}

Volume 18, numéro 1-2, printemps-été 2012

Les administrations publiques à l'ère du numérique

URI : https://id.erudit.org/iderudit/1009259ar

DOI : https://doi.org/10.7202/1009259ar

Aller au sommaire du numéro

Éditeur(s)

L'Observatoire de l'administration publique

ISSN

1203-3294 (imprimé)

1929-3348 (numérique)

Découvrir la revue

Citer cet article

Blum, G. (2012). De la liberté du logiciel et de son ouverture : tour d'horizon et perspectives. Télescope, 18(1-2), 121-138. https://doi.org/10.7202/1009259ar
Résumé de l'article

Dans cet article, l'auteur présente ce qu'est le logiciel libre et ce qu'il représente. Pour ce faire, il s'intéresse à différents éléments, tels que les concepteurs, les utilisateurs, le mécanisme de fonctionnement et les raisons poussant les individus à recourir à ce type de logiciel. Une fois l'objet défini et bien décrit, il examine les conséquences du logiciel libre dans d'autres domaines et relève les enjeux en matière d'administration publique. La dernière partie revient sur l'état d'avancement du logiciel libre au Québec et dans l'administration publique québécoise. 


\title{
DE LA LIBERTÉ DU LOGICIEL ET DE SON OUVERTURE : TOUR D'HORIZON ET PERSPECTIVES
}

Par Guillaume Blum, Chargé de cours, Département de management et technologie, Université du Québec à Montréal • guillaume@blum.qc.com

\begin{abstract}
RÉSUMÉ Dans cet article, l'auteur présente ce qu'est le logiciel libre et ce qu'il représente. Pour ce faire, il s'intéresse à différents éléments, tels que les concepteurs, les utilisateurs, le mécanisme de fonctionnement et les raisons poussant les individus à recourir à ce type de logiciel. Une fois l'objet défini et bien décrit, il examine les conséquences du logiciel libre dans d'autres domaines et relève les enjeux en matière d'administration publique. La dernière partie revient sur l'état d'avancement du logiciel libre au Québec et dans l'administration publique québécoise.
\end{abstract}

\begin{abstract}
In this article, the author describes and explains open source software and what it represents. To this end, he examines various elements - such as designers, users, operating mechanism, etc. - and the reasons why people have turned to this type of software. Having defined and fully described the object of his research, he discusses the consequences of open source software in other fields and the issues that its use raises for public administrations. The last section of his article reviews the status of open source software in Quebec and in the province's public administration.
\end{abstract}

Pour citer cet article : Blum, G. (2012). « De la liberté du logiciel et de son ouverture : tour d'horizon et perspectives », Télescope, vol. 18, n 1-2, p. 121-138.

D epuis quelques années, nombre de professionnels œuvrant dans les organisations, qu'elles soient publiques ou privées, ont eu l'occasion de voir apparaître un nouveau terme - pour ne pas dire concept - dans le vocabulaire informatique : celui de logiciel libre ou de logiciel à code source ouvert (logiciel open source en anglais). Tant le terme que le type de logiciel se répandent dans le monde professionnel, et ce, depuis suffisamment d'années pour qu'il ne s'agisse pas d'une mode comme on en voit tant dans nos univers organisés.

D'abord apparu dans le milieu de la recherche, dans les secteurs associatifs et comme hobby, le logiciel libre se diffuse lentement dans le monde des organisations et des administrations. Certains pensent, à l'instar de Di Cosmo (2002), que les administrations publiques devraient, par la nature même de leurs activités, utiliser des logiciels libres. D'autres estiment qu'il peut y avoir des avantages économiques associés à une telle utilisation, et que l'on doit passer d'une économie de rente basée sur la vente du logiciel à une économie de service - par exemple, le support, le codage d'une nouvelle fonctionnalité, etc. (Weber, 2004). Quelques-uns en font un combat politique et juridique afin de modifier les pratiques informatiques en matière gouvernementale. Le procès gagné le 3 juin 2010 en Cour supérieure du Québec par Solution Linux contre la Régie des rentes du Québec, qui a donné naissance en 2011 à l'obligation de la prise en considération du logiciel libre dans la nouvelle Loi sur la gouvernance et la gestion des ressources informationnelles 
des organismes publics et des entreprises du gouvernement ${ }^{1}$, en est un exemple. D'autres acteurs continuent de lutter férocement contre.

L'objectif de cet article est de déterminer ce qui se cache derrière les termes " logiciel libre " ou " logiciel à code source ouvert " et de chercher à comprendre ce que ce type de logiciel peut ou va modifier à nos modes d'organisation à travers l'extension du mouvement du libre. Pour ce faire, la première partie présente un tour d'horizon des idées et des pratiques les plus significatives au moyen de cinq questions. La deuxième partie illustre l'extension du mouvement du libre à d'autres domaines que celui purement logiciel et la dernière observe les pratiques et les tendances au Québec.

\section{- LE LOGICIEL LIBRE À TRAVERS CINQ QUESTIONS}

Cette section vise à dresser un portrait du logiciel libre en répondant à cinq questions.

\section{Qu'est-ce que le logiciel libre?}

Il s'avère nécessaire d'amorcer cette section en définissant le « logiciel libre ». Selon la Free Software Foundation (2004), pour qu'un logiciel soit considéré comme " libre ", il doit respecter certaines propriétés minimales. Il s'agit des quatre libertés suivantes : celle d'exécuter le programme quel qu'en soit l'usage, celle de pouvoir étudier le fonctionnement du programme et éventuellement de l'adapter à son propre usage, ce qui nécessite l'accès au code source du programme, celle de redistribuer des copies à d'autres et celle d'améliorer le programme et de distribuer ces modifications. Ainsi, un logiciel libre correspond à un logiciel que l'on peut utiliser, dont le code source est accessible, que tout le monde peut modifier et redistribuer, sans qu'une tierce personne cherche à bloquer cela. Pour ce faire, le logiciel libre s'appuie sur la notion de gauche d'auteur (voir plus loin).

Cette définition est très proche du logiciel que l'on qualifie d'open source ou à code source ouvert, qui se doit - pour être qualifié comme tel - d'être librement redistribuable, d'inclure le code source, d'autoriser les travaux dérivés, de respecter l'intégrité de l'auteur du code source, de ne pas discriminer des personnes ou des groupes, de ne pas discriminer des champs de préoccupation, d'attacher la licence au logiciel, de ne pas être spécifique à une partie du logiciel, de ne pas restreindre d'autres logiciels, d'être technologiquement neutre (Open Source Initiative, 2007). Ainsi, la plupart du temps, un logiciel libre et un logiciel à code source ouvert correspondent au même objet technique - pour peu que l'on puisse qualifier un logiciel d'objet.

Toutefois, une distinction de taille différencie le logiciel libre du logiciel à code source ouvert : il s'agit du but poursuivi. Le logiciel libre vise un objectif éthique, celui de donner aux programmeurs et aux utilisateurs une liberté - la plus grande possible - face à l'outil informatique. Il s'agit très clairement d'afficher que

L.R.Q., chapitre G-1.03. 
l'usage de ce type de logiciels ne doit pas être restreint par quelque personne ni pour quelque raison que ce soit, à partir du moment où l'on respecte les quatre libertés fondamentales énoncées plus haut. Il est amusant de voir que cette situation peut aboutir à d'étranges paradoxes, comme l'usage par l'armée française du logiciel SPIP, logiciel créé par des militants très ancrés à gauche et à tendance libertaire et désapprouvant l'armée, mais souhaitant néanmoins que le code de leur logiciel puisse être utilisé par elle (Demazière, Horn et Zune, 2006).

Le logiciel à code source ouvert vise un tout autre objectif. Il s'agit ici d'un mode de diffusion du logiciel visant à la plus grande efficacité possible en termes d'utilisation économique et d'innovation. En effet, si un grand nombre de personnes contribuent à un logiciel à code source ouvert, celui-ci bénéficiera de l'apport de ces dizaines, centaines ou même milliers d'utilisateurs. Aussi, l'effort et le coût pour chaque individu seront minimes par rapport au bénéfice lié à l'utilisation. Il s'agit ici d'une mise en commun afin de minimiser l'effort et le coût individuels. Par ailleurs, reposant sur la participation et les idées de tous, les modes de développement à code source ouvert peuvent favoriser la transformation des idées les plus intéressantes en innovations, en plaçant ces idées en compétition ou plus précisément en coopétition, c'est-à-dire dans un contexte où des acteurs se trouvent à la fois à coopérer et à compétitionner (Prévot, 2007). Ainsi, dans le cadre de développement du noyau Linux, il arrive souvent que plusieurs algorithmes soient dans cette position de forme de " compétition amicale ", avec pour objectif d'obtenir la meilleure solution technique et d'accélérer la transformation de l'idée en code propre et utilisable. La situation des algorithmes d'ordonnancement (une partie importante du noyau) est à ce titre exemplaire : l'algorithme utilisé a été plusieurs fois modifié suivant les progrès de chacun et l'impact sur les performances.

Le logiciel libre adopte donc une perspective d'ordre idéologique et éthique, là où le logiciel à code source ouvert renvoie à des arguments d'ordre économique et technique poursuivant la plus grande efficience possible. Bien que les objectifs soient clairement différenciés, d'un point de vue technique ou légal si un logiciel libre ou à code source ouvert respecte l'une des définitions, il respecte également l'autre dans la plupart des cas. Ce cas est particulièrement intéressant. À quelques exceptions près, un logiciel libre est également un logiciel à code source ouvert.

Ceci n'est pas sans incidence, car c'est sans doute là l'origine du succès de ce type de logiciel (Blum, 2008). S'ils n'avaient pas été efficaces, les logiciels libres n'auraient jamais dépassé le groupe des quelques hackers les développant à travers le monde. En revanche, s'ils n'avaient pas visé une perspective idéologique et éthique, notamment à leurs débuts, ils n'auraient pas su - notamment à l'époque où ils étaient encore marginaux - aller chercher suffisamment de développeurs pour participer à des projets informatiques qui paraissaient a priori fous et irréalistes. Par ailleurs, les différences de perspectives des auteurs de ces logiciels sont une source de diversité, ce qui alimente non seulement les débats passionnés dans les communautés de développeurs, mais représente aussi une formidable source d'innovation dans une perspective dialectique (Blum et Ebrahimi, 2007a). 


\section{Où trouve-t-on les logiciels libres?}

Nous avons précédemment évoqué le succès du logiciel libre. Mais peut-on réellement parler d'un succès? Finalement, qui utilise les logiciels libres? À première vue, peu de gens ont recours à ce type de logiciels. Par conséquent, la réponse - du moins en termes de diffusion ${ }^{2}$ - serait négative. Toutefois, à y regarder de plus près, la plupart des utilisateurs des technologies des dernières années utilisent de tels logiciels. En effet, tel Monsieur Jourdain qui faisait de la prose sans le savoir, nous sommes presque tous utilisateurs indirects de logiciels libres, lesquels ont infiltré l'infrastructure informatique et électronique de nos sociétés modernes. Nombre de téléphones portables, d'appareils photo, de lecteurs de musique, de téléviseurs, de serveurs, d'appareils électroménagers, de voitures, d'avions, etc. utilisent des logiciels libres. Ainsi, l'infrastructure - invisible - s'appuie grandement sur ce type de logiciels du fait de leur flexibilité et efficacité. Près de $80 \%$ des serveurs Internet fonctionnent sur des logiciels libres (Netcraft, 2011), et même des systèmes relativement fermés s'appuient sur de tels logiciels, par exemple le système d'exploitation des ordinateurs et appareils mobiles d'Apple, reposant notamment sur le logiciel libre FreeBSD (Apple, 2011).

Ainsi, le nombre de logiciels et de projets libres a considérablement augmenté au cours des dernières années. SourceForge, hébergeur de projets libres et ouverts, a vu le nombre de projets passer de près de 125000 en 2006 à plus de 260000 en 2010, avec près de 3 millions de développeurs (Sourceforge, 2011).

Finalement, l'univers informatique repose en grande partie sur les logiciels libres, que de très nombreuses organisations utilisent, à l'exception du bureau de chaque utilisateur. Le marché des systèmes d'exploitation d'ordinateur ainsi que la plupart des logiciels de bureautique demeurent majoritairement fermés. Si historiquement des raisons ergonomiques expliquaient cette non-percée dans le secteur des utilisateurs finaux et non spécialistes, cette explication est aujourd'hui largement bancale, car d'énormes progrès ont été réalisés au cours des dernières années. L'explication se trouve plutôt du côté de la puissance marketing dont font preuve les sociétés commerciales vendant des logiciels fermés, ainsi que dans un attachement à un ensemble de logiciels déjà maîtrisés, rendant difficile tout changement, tant en termes cognitifs qu'en termes de coût de formation lié à l'utilisation de nouveaux outils. Est-ce à penser que le bureau de l'utilisateur ne sera jamais libre? Cela reste incertain, puisqu'un mouvement massif vers l'informatique en nuage (cloud computing ou infonuagique) est en train de recomposer la structure des systèmes informatiques.

2 Le succès ne se mesure pas nécessairement en nombre d'utilisateurs. Par exemple, pour un utilisateur, il peut être plus important d'avoir un système totalement libre, ou offrant plus de possibilités techniques. Toutefois, dans le cas du logiciel libre où les utilisateurs sont aussi les concepteurs du système, augmenter le nombre d'utilisateurs n'est pas sans incidence sur le nombre de fonctionnalités ou le rythme du progrès des logiciels ou des systèmes informatiques. 


\section{Qui sont les concepteurs des logiciels libres?}

Nous avons traité jusqu'ici des logiciels libres sans nous pencher sur les concepteurs, mis à part le constat que tout le monde pouvait contribuer. Or pouvoir contribuer n'est pas synonyme de contribuer, et avec l'accroissement du nombre d'utilisateurs, on note qu'une part de plus en plus importante de ces derniers ne sont plus des utilisateurs-contributeurs, ce qui n'est pas sans poser des problèmes de transformation des communautés de hackers originaux, devant modifier leurs habitudes (Jullien et Zimmermann, 2006).

Historiquement, les utilisateurs-développeurs de logiciels libres étaient des hackers. Ce terme mérite d'être défini, car il est souvent mal compris et mal utilisé. Dans la représentation commune, le hacker serait un pirate informatique cherchant à s'introduire dans des systèmes informatiques ou à les casser. Il s'agit là plutôt de la définition d'un cracker, ou pirate, c'est-à-dire un hacker mal intentionné. S'il arrive à des hackers (dans le sens propre du terme) de s'introduire dans des systèmes, c'est généralement pour aider les développeurs ou le mainteneur desdits systèmes. On peut donner au terme hacker la définition suivante : il s'agit d'une personne qui cherche à comprendre le fonctionnement d'un outil ou d'un système technique en empruntant des chemins détournés comme le bidouillage et la manipulation en vue de parfaire sa maîtrise et d'arriver à de nouveaux usages initialement non prévus. Les hackers ont coutume de dire qu'il s'agit plus d'une mentalité que de compétences spécifiques. Ainsi, l'amateur démontant sa voiture pour la comprendre et la modifier peut être considéré comme un hacker. Son objectif n'est pas de faire le mal, mais de parfaire sa compréhension du système technique. D'une certaine façon, cette perspective visant la maîtrise des systèmes techniques est une forme de lutte contre l'aliénation à leur égard (Blum, 2008; Ellul, 1990).

L'histoire des systèmes techniques doit beaucoup aux hackers. Les frères Wright, lorsqu'ils créèrent le premier engin volant plus lourd que l'air au début du XX e siècle, peuvent être considérés comme des hackers à l'instar de tous les autres amateurs de l'époque qui cherchaient à construire une machine volante. L'informatique n'échappe pas à cette histoire. Dans les années 1970, les premiers microordinateurs individuels étaient vendus en kit et l'utilisateur devait commencer par le montage matériel du système lors de sa réception. C'est la raison pour laquelle l'industrie informatique est née, selon la tradition, dans le garage de jeunes gens ${ }^{3}$.

Le parallèle ne s'arrête pas là. Comme pour l'aéronautique et toutes les autres sciences techniques, nous avons tendance à mettre de l'avant les compétences et les progrès individuels au détriment de ceux des communautés. Dans le domaine aéronautique, un grand nombre de passionnés travaillaient partout dans le monde à la réalisation de l'avion. Ceux-ci, bien que concurrents, étaient également en correspondance et profitaient des avancés des uns et des autres. Si les frères Wright

3 Citons les jeunes Steve Wozniak, Steve Jobs, Bill Gates, pour n'en prendre que trois, tous très jeunes, passionnés et sans profession, pouvant ainsi passer tout leur temps à pratiquer leur hobby à partir duquel ils ont fait leur métier et créé des empires industriels (Apple pour les deux premiers, Microsoft pour le troisième). 
sont les premiers à avoir fait voler un avion, leur travail aurait été impossible sans les centaines d'autres passionnés travaillant sur le même projet. De la même façon, les passionnés d'informatique se regroupaient et pouvaient échanger, notamment dans certaines universités américaines et dans des territoires où nombre de passionnés se retrouvaient. De là sont nées la Silicon Valley contemporaine et la route 128. Ces communautés ont aussi été les premières à utiliser l'informatique pour communiquer grâce à Arpanet, l'ancêtre d'Internet, et plus tard Internet, et ainsi à créer les premières communautés en ligne. Des hackers de partout dans le monde s'y regroupaient sur des projets communs.

La plupart des logiciels libres sont ainsi des projets collectifs nés au sein de communautés. L'un des plus importants est le projet GNU, projet initié par Richard Stallman, à l'origine du concept de logiciel libre et de la notion de gauche d'auteur, visant à établir sous licence libre un système d'exploitation complet (Williams, Stallman et Musutti, 2010). Ce projet fut complété par le noyau Linux, dernière pièce du puzzle complexe que représente un système d'exploitation au début des années 1990. De la combinaison des deux est né le système GNU/Linux, à la base de nombreux systèmes d'exploitation libres contemporains (par exemple, Red Hat, Debian, Ubuntu, Jolicloud, Android, etc.).

Un autre projet important placé sous licence libre plutôt que sous le régime traditionnel de la propriété intellectuelle est le World Wide Web, plus communément appelé le Web, partie constituante d'Internet utilisant un langage d'hypertexte (HTML pour HyperText Markup Language) et un protocole d'accès (HTTP pour HyperText Transfer Protocol) qui ont permis l'explosion d'Internet auprès du grand public. Si Tim Berners-Lee - le créateur du Web - n'avait pas rendu libres de droits les technologies qu'il développa, le Web et Internet n'auraient probablement pas obtenu le succès qu'ils ont connu ou, du moins, beaucoup moins rapidement.

Ces communautés virtuelles de développeurs et de hackers sont toujours à l'avant-garde des technologies de l'informatique. Elles possèdent leurs propres codes et ont fait l'objet de recherche d'ordre ethnographique (voir par exemple Blum et Ebrahimi, 2007b).

\section{Comment fonctionne le gauche d'auteur ou copyleft?}

Il serait maintenant opportun de savoir comment tout cela fonctionne. Sur quel mécanisme - légal - le logiciel libre s'appuie-t-il? La réponse est sur une innovation sociale, à savoir les licences libres favorisant le gauche d'auteur (copyleft) qui est en fait un mécanisme reposant sur le droit d'auteur pour le détourner de sa finalité première. À la base d'un logiciel, on trouve le code, écrit dans un langage compréhensible par des êtres humains (les programmeurs) et transformé ou interprété par la machine pour être exécuté. Comme toute œuvre de l'esprit, ce code est protégé par la propriété intellectuelle, et puisqu'il s'agit de langage, c'est le droit 
d'auteur qui s'applique - ou du moins qui devrait s'appliquer ${ }^{4}$. L'idée est donc, en s'appuyant sur le droit d'auteur, d'ajouter une clause dans la licence précisant les quatre libertés fondamentales énoncées précédemment. La plus connue de ces licences est la licence GPL (General Public Licence), mais il en existe plusieurs autres (LGPL, GFDL, CeCILL, CC-by-SA, etc. ${ }^{5}$ ).

La particularité des licences de gauche d'auteur est leur viralité, ou potentiel de diffusion. Le principe est simple : toute œuvre placée sous licence de gauche d'auteur est libre, mais les modifications ultérieures doivent elles aussi être placées sous la licence initiale. Un utilisateur de logiciel libre sous gauche d'auteur souhaitant ajouter des caractéristiques puis redistribuer son travail doit le faire selon les mêmes conditions, à défaut de quoi il ne peut pas utiliser le code du logiciel sous copyleft. En adoptant ce mode de diffusion, on se protège du phénomène que les économistes nomment " passager clandestin " : un individu ou une organisation s'approprie le travail d'autrui pour le modifier et en tirer profit sans participer à l'effort collectif (Ordoneau, 2011).

\section{Pourquoi utiliser le logiciel libre?}

Pour achever ce tour d'horizon du concept de logiciel libre, il convient de se pencher sur la question du pourquoi ou sur l'idéologie promue par le logiciel libre. Le terme " idéologie " est utilisé pour désigner un ensemble cohérent d'idées, représentant un système-monde, c'est-à-dire une représentation cognitive du monde de la part d'un acteur ou d'un ensemble d'acteurs (Solé, 2000). Nous cherchons donc dans cette section à présenter le système de pensées et de valeurs promu par les tenants du logiciel libre, qui s'est par la suite retrouvé dans d'autres domaines comme nous le verrons dans la section suivante.

L'idée de liberté, telle qu'elle est exprimée dans le logiciel libre, prend racine dans la révolution américaine de 1776. Elle est déterminante pour les communautés du libre : " La culture de travail de la communauté de développement du logiciel libre engendre chez les contributeurs un esprit-de-corps [en français dans le texte] qui perpétue la communauté » (Elliott, 2003, p. 45, traduction libre). Pour Linus Torvalds, programmeur à l'origine du noyau Linux et encore aujourd'hui architecte principal de celui-ci, la source de motivation des hackers est le plaisir, l'ordinateur étant source de plaisir. Il décrit le phénomène comme un nouveau mode

4 En effet, dans les années 1980 les États-Unis ont commencé à accepter des brevets pour protéger du code informatique, ce qui est extrêmement problématique, car le brevet est censé protéger l'application d'une idée et non l'idée elle-même. Le problème du logiciel est que l'idée elle-même et son application ne font qu'un à travers son écriture dans un langage informatique. En permettant le brevet, on permet le dépôt direct d'une idée, voire d'idées très simples, bloquant l'innovation, car transformant le travail des développeurs en spécialistes du droit de la propriété intellectuelle visant à rechercher les brevets existant pour ensuite chercher à les contourner. On transforme ainsi un outil qui vise à favoriser l'innovation en véritable champ de mines empêchant l'innovation pour qui n'a pas les moyens de jouer dans ce jeu associant compétences informatiques, compétences juridiques et portefeuille de brevets.

5 II existe aussi des licences libres non gauche d'auteur (BSD, CC-by, X11, etc.). 
de rapport au monde s'opposant à la conception protestante du travail comme une fin en soi qui justifie le capitalisme et l'accumulation de profit. Himanen (2001) décrit l'émergence d'une éthique hacker basée sur trois pôles : (1) le travail est vu comme un jeu, une passion; (2) l'argent est moins important que le partage; (3) le réseau rend la structure plate.

Cette idée d'appartenance à une communauté dans laquelle on fait don de son temps et de son travail pousse certains à dire qu'il s'agit d'un mode de régulation basé sur une économie du don, c'est-à-dire un espace où le statut social n'est pas déterminé par ce que l'on contrôle, mais par ce que l'on donne (Raymond, 1999). Cette conception repose sur l'idée de la suppression de la rareté dans l'univers informatique, d'une abondance en matière d'espace disque, de réseau, de bande passante, de puissance de calcul. Toutefois, la rareté n'a pas disparu, elle s'est déplacée vers l'individu rendant son temps et ses ressources cognitives rares, ce qui a été théorisé par l'économie de l'attention (Davenport et Beck, 2001) et a amené la recherche à s'intéresser plus spécialement à l'économie de la contribution, s'intégrant dans trois champs d'investigation : le domaine numérique, les systèmes locaux d'innovation et l'économie sociale et solidaire (Beraud et Cormerais, 2011).

Cet attachement à la contribution dans le domaine numérique peut s'expliquer par trois types de motivation : le militantisme politique, l'attrait technologique et le gain de réputation dans une communauté lié ou non à une possibilité d'affaires (Demazière, Horn et Zune, 2006). Il est également possible de voir dans la contribution au logiciel libre une forme de contre-réaction à l'intégration dans un système capitaliste de type financier, ce qu'Auray et Vicente (2006) qualifient de réaction au "moment intégré ", ce troisième temps du mouvement informatique est apparu avec Internet, après le " moment centralisé " (de 1950 au milieu des années 1970) et le "moment déconcentré " (du milieu des années 1970 au milieu des années 1990). Cela place les acteurs à la fois dans l'action et dans une quête existentielle allant au-delà du travail. Pour les individus contribuant aux projets libres étudiés par ces auteurs (Debian et OpenBSD), l'engagement dans le logiciel libre correspond " à un désintéressement ou une déception vis-à-vis de l'activité professionnelle actuelle, sur laquelle les participants n'hésitent pas à émettre des critiques virulentes [...] majoritairement sur quatre points : la temporalité, la proximité, la qualité et le rapport à l'objet produit » (Auray et Vicente, 2006, p. 5). Ainsi, l'une des grandes forces du logiciel libre est d'engendrer des incitations de deux ordres : (1) une incitation collective, l'effort collectif d'amélioration entraîne pour chacun une externalité d'usage; (2) des incitations individuelles, les contributeurs profitant des effets d'apprentissage (par la pratique du codage et par la lecture du code d'un autre) et de réputation, entraînant une forme de reconnaissance par les pairs (Foray et Zimmermann, 2001).

D'autres auteurs se sont attachés à étudier et à décrire les communautés libres de l'intérieur, en se basant sur une approche ethnographique. Ainsi, Stewart et Gosain (2001) observent les communautés libres et les décrivent comme unifiées par une idéologie cohérente composée de normes, de croyances et de valeurs interreliées, comme l'illustre le tableau 1. Cette idéologie a pour effet d'entraîner 
un mode de fonctionnement particulièrement efficace au sein de la communauté (Stewart et Gosain, 2006). À partir d'une autre ethnographie, dans la communauté Ubuntu, Blum et Ebrahimi (2007b) ont montré l'attachement au bidouillage, à la liberté, au partage, à l'autodérision, à l'expertise technique et à la démocratie.

\section{TABLEAU 1 : LES NORMES, LES VALEURS ET LES CROYANCES À LA BASE DE L'IDÉOLOGIE DANS LES COMMUNAUTÉS LIBRES}

\begin{tabular}{|c|c|}
\hline Normes & $\begin{array}{l}\text { - Faire un fork } 6 \text { est tabou. } \\
\text { - Distribuer des changements sans la coopération des modérateurs ne se } \\
\text { fait pas. } \\
\text { - Enlever le nom d'une personne de l'historique d'un projet, des } \\
\text { remerciements ou de la liste des mainteneurs ne se fait pas sans un } \\
\text { consentement explicite. }\end{array}$ \\
\hline Valeurs & $\begin{array}{l}\text { - Les meilleurs bidouilleurs gagnent. } \\
\text { - Toute l'information doit être libre. } \\
\text { - Vous n'êtes un hacker que lorsque d'autres hackers vous qualifient } \\
\text { comme tel. } \\
\text { - Les extensions non triviales de fonction sont meilleures que des patches } \\
\text { et le débogage. }\end{array}$ \\
\hline Croyances & $\begin{array}{l}\text { - Ce qui marche dans une grande distribution est mieux que ce qui ne } \\
\text { marche pas. } \\
\text { - Avec suffisamment d'yeux, tous les bogues seront repérés. } \\
\text { - La pratique est meilleure que la théorie. }\end{array}$ \\
\hline
\end{tabular}

Source : d'après Stewart et Gosain, 2001.

\section{- DES CONSÉQUENCES DU LOGICIEL LIBRE : LA CONTAGION À D'AUTRES DOMAINES À TRAVERS LE MOUVEMENT DU LIBRE}

Si l'idée de liberté de l'information et de licence libre est d'abord née avec le logiciel, elle s'est étendue par la suite à d'autres domaines. Citons comme exemple Wikipédia, dont l'idée est de rendre la connaissance libre au moyen d'une encyclopédie écrite collectivement selon le principe de la contribution :

La philosophie du « libre " étant au cœur du projet, nous sommes particulièrement attachés aux possibilités de redistribuer le plus librement possible la plus grande partie possible du contenu de Wikipédia, de sorte que les documents originaux publiés dans cet esprit soient toujours préférés à ceux placés sous une licence non libre (Wikipédia, 2011).

En fait, les contenus non libres représentent essentiellement des documents audio, photo ou vidéo. Ainsi, la quasi-totalité de Wikipédia se trouve sous la licence Creative Commons (voir tableau 2). Plus globalement, le terme souvent utilisé

${ }_{6}$ Un fork est la reprise d'un projet déjà existant, et continuant d'exister, par d'autres personnes que les initiateurs du projet. Cela est possible par l'accès au code source. 
de Web 2.0 reprend cette idée de contribution et d'appropriation collective pour une plus grande diffusion.

Les licences Creative Commons initiées par Lawrence Lessig dans les années 1990 cherchent à étendre aux autres domaines issus du savoir les principes des licences des logiciels libres. Elles peuvent donc s'appliquer à toutes les œuvres de l'esprit : textes, musiques, vidéos, photographies, plans, etc. Elles présentent la spécificité d'être modulaires pour correspondre aux attentes particulières des auteurs. Six licences disposent de propriétés différentes regroupées dans le tableau qui suit.

\section{TABLEAU 2 : LES LICENCES CREATIVE COMMONS}

\begin{tabular}{|l|l|l|}
\hline TYPE DE LICENCE & PROPRIÉTÉS & LICENCE LIBRE \\
\hline CC-by & Paternité & Libre \\
\hline CC-by-sa & Paternité, partage des conditions initiales à & Libre avec gauche \\
CC-by-nd & I'identique & d'auteur \\
\hline CC-by-nc & Paternité, pas de modification & Non libre \\
\hline CC-by-nc-sa & Paternité, pas d'utilisation commerciale & Non libre \\
\hline CC-by-nc-nd & Partage des conditions initiales à l'identique & Non libre \\
\hline
\end{tabular}

L'encyclopédie libre ne constitue pas une exception à l'extension du domaine du libre. De plus en plus d'artistes placent leurs travaux sous des licences libres, et ce, que leur motivation soit de toucher un plus vaste public ou de réagir à la marchandisation de la culture. Des ingénieurs ou des chercheurs partagent également sous de telles licences les plans et les travaux décrivant leurs inventions ou leurs découvertes. Au demeurant, au cours des dix dernières années, plusieurs revues scientifiques sont passées sous licences libres d'accès. Citons à titre d'exemple la très prestigieuse revue PloS One. Cette nouvelle réalité influence l'organisation même du travail. Par exemple, dans le champ de la biologie, de plus en plus de travaux se font dans le domaine de la sélection assistée par marqueur, où l'on vise à repérer sur le génome des marqueurs de caractéristiques et à les partager avec la communauté scientifique pour être capable par la suite de connaître a priori les propriétés d'un croisement selon les attentes du génie biologique. Il s'agit, en quelque sorte, d'une version propre et libre des organismes génétiquement modifiés.

Ces extensions touchent encore bien d'autres domaines. Dans le journalisme, on citera l'exemple de Wikileaks qui s'est entre autres illustré depuis 2006 avec la sortie de documents traitant d'assassinats en Somalie, de corruption au Kenya ou dans des banques occidentales, de négociations secrètes entre des gouvernements dans le cadre de l'Accord commercial relatif à la contrefaçon (ACRC, mais plus connu sous son acronyme anglais ACTA - Anti-Counterfeiting Trade Agreement) et, bien sûr, des war logs iraquiens et des câbles diplomatiques américains (aussi 
nommés " cablegate ") qui ont beaucoup fait parler d'eux (sur ce sujet voir Deharo et Dimitrova, 2011).

Du côté des administrations publiques, outre le passage aux logiciels libres, on observe l'effet de ce courant dans deux domaines : le passage au Web 2.0 et le mouvement d'ouverture des données. Un gouvernement utilisant les principes du Web 2.0 poursuit l'objectif d'améliorer le lien avec ses citoyens en offrant non seulement un certain nombre de services en ligne, mais aussi en donnant à la population la possibilité de faire remonter de l'information, des critiques ou des idées jusqu'au gouvernement. Il s'agit de mettre en place l'infrastructure technologique pour permettre à tous de s'exprimer. L'ouverture des données consiste à partager des données non confidentielles en les diffusant sous une licence libre afin qu'elles puissent être utilisées à une fin initialement non prévue. S'il s'agit d'une politique gouvernementale (municipale) favorisant cette pratique au sein de ses administrations, on parle de " gouvernement " ouvert ou de "Ville ouverte ". Plusieurs exemples peuvent illustrer l'intérêt de ce concept.

En France, l'organisme sans but lucratif Regards Citoyens s'est associé à Transparence International France pour constituer, à partir de documents publics, une base de données des auditions par les députés de l'Assemblée nationale entre juillet 2007 et 2010 afin de réaliser une cartographie du lobbying (Regard Citoyen, 2011).

En Grande-Bretagne, le site Internet openlylocal.com permet aux citoyens de retrouver sous une forme structurée un ensemble de données concernant les communes, comme le nom et l'historique des maires, le nombre d'habitants, l'évolution démographique ou encore les dépenses de la commune. Grâce à un ensemble d'outils, tout citoyen peut mener des comparaisons statistiques entre les communes.

Aux États-Unis, le site Internet crimemapping.com permet de visualiser sur une carte géographique les crimes et les délits commis. Cette visualisation est possible à l'aide d'information de source policière. Toujours aux États-Unis, la ville de Zanesville (Ohio) a été condamnée à une amende de 10,9 millions de dollars à la suite de la démonstration d'une discrimination raciale concernant le raccord des foyers au réseau d'eau courante de la ville. La démonstration repose sur le regroupement et l'analyse de données et leur représentation géographique, montrant un très fort niveau de corrélation entre les communautés ethniques des quartiers et la distribution (ou non) de l'eau (Ludlow, 2008).

Plusieurs villes et États ont commencé à diffuser des données publiques. Une recherche non exhaustive permet d'identifier parmi les villes ou autres instances territoriales les plus avancées dans le mouvement d'ouverture des données Montpellier, Paris, Rennes, Bordeaux, New York City, San Francisco, Boston, Chicago, La Nouvelle-Orléans, Portland, Seattle, City of Ann Arbor, Londres, Birmingham, Lichfield, Warwickshire, Kent, Victoria (Australie), Nouvelle-Galles du Sud. Au Canada, on trouve Vancouver, Nanaimo, Toronto, Calgary, Edmonton, Mississauga, Ottawa, London et Windsor. Quant aux États, les plus avancés sont les États-Unis, la Californie, le Maine, le Michigan, le Massachusetts, le Rhode Island, 
l'Utah, le Royaume-Uni, l'Estonie, la Finlande, le Danemark, l'Espagne, la Russie, le Kenya, l'Australie, le Canada et la Colombie-Britannique.

Ces différentes pratiques dans le monde du logiciel ou dans les autres secteurs d'activité ont un impact fort sur le monde du travail, notamment sur la logique d'organisation. Il est par exemple difficile de conjuguer une conception pyramidale de l'organisation avec la structure en réseau des projets initiatives libres (Von Hippel, 2005), ou encore d'associer culte du secret et volonté de transparence et d'ouverture, ou logique basée sur les produits avec logique basée sur les services. Cela donne parfois naissance à des conflits qui tirent leurs origines de conceptions idéologiques différentes, lorsque la culture organisationnelle ne suit pas cette tendance. C'est en cela que l'on peut parler de mouvement du libre.

\section{- LE LOGICIEL LIBRE AU QUÉBEC}

Les premières initiatives liées au logiciel libre existent au Québec depuis les années 1990. Le groupe d'utilisateurs Linux-Québec, fondé en septembre 1997, est considéré comme l'une des premières associations d'utilisateurs dans ce domaine (Couture et autres, 2010). Douze autres groupes d'utilisateurs de Linux (GUL) existent depuis les années 2000 (Association francophone des utilisateurs de logiciels libres, 2011). Les différentes activités liées au monde du logiciel libre sont présentées sur l'Agenda du libre au Québec ${ }^{7}$. On y apprend que près de la moitié des événements se déroulent dans la région de Montréal.

Plusieurs organismes et associations existent également : FACIL, pour l'appropriation collective de l'informatique libre (FACIL), l'Association professionnelle des entreprises en logiciels libres (APELL) qui regroupe dix-sept entreprises spécialisées en logiciels libres. On trouve également plusieurs groupes communautaires, dont Communautique, Île sans fil, Espaces Temps Montréal. Dans les universités, Daniel Pascot organise un cours portant sur les logiciels libres à l'Université Laval. Sur le plan de la recherche, le Laboratoire de communication médiatisée par ordinateur (LabCMO) mène divers travaux et a organisé plusieurs colloques. On trouve également la Chaire de logiciel libre, Finance sociale et solidaire. Plusieurs chercheurs conduisent également des recherches sur le logiciel libre dans les universités québécoises.

Dans leur note de recherche, Couture et ses collègues (2010) recensent au Québec, pour l'année 2008, 61 projets de développement de logiciels, 58 activités d'éducation et de promotion des logiciels libres, 23 types d'usage à travers des migrations, l'administration de serveurs ou l'utilisation qualifiée de " simple ". Ils comptent également, pour la même année, 52 groupes sociaux ou activités permanentes traitant de logiciels libres au Québec (voir annexe 1), qui représentent des groupes constitués ou non, poursuivant ou non un objectif lucratif, mais ayant une base " permanente ", donc participant à l'organisation d'une conférence ou

7 Pour connaître les diverses activités, voir www.agendadulibre.qc.ca. 
d'événements de manière récurrente. Depuis 2010, un événement d'importance, le Salon du logiciel libre du Québec, est organisé annuellement ${ }^{8}$.

Malgré cette profusion de petites organisations confirmant l'existence d'un écosystème favorable, le gouvernement du Québec est peu avancé sur la question des logiciels libres, ceux-ci représentant encore dans plusieurs administrations une question taboue. Malgré l'existence de quelques projets très novateurs - comme le projet de Centre 911 basé sur des logiciels libres mené par le ministère de la Sécurité publique du Québec qui sert de référence mondiale sur la question -, l'administration publique québécoise fait figure de parent pauvre sur cette thématique du logiciel libre. Mais les choses changent. Depuis la Loi sur la gouvernance et la gestion des ressources informationnelles des organismes publics et des entreprises du gouvernement ${ }^{9}$, le logiciel libre commence à prendre de l'importance dans la stratégie technologique et informationnelle du gouvernement du Québec. En effet, le dirigeant principal de l'information, les dirigeants réseau de l'information (DRI santé et DRI éducation), les dirigeants sectoriels de l'information (DSI des organisations publiques) doivent " prendre les mesures requises pour que les organismes publics considèrent les logiciels libres au même titre que les autres logiciels ». Par ailleurs, le Conseil du trésor " peut également déterminer des orientations portant sur les principes ou les pratiques à favoriser en matière de gestion des ressources informationnelles, incluant la nécessité de considérer les logiciels libres au même titre que tout autre logiciel, qui serviront de référence aux organismes publics ». Pour accompagner ce projet de loi, le Centre de services partagés du Québec a créé, via le Centre de leadership et de développement des compétences, une série de huit cours portant sur différents enjeux liés au logiciel libre : les aspects stratégiques; les technologies; la production; l'utilisation gouvernementale; la sécurité; les aspects financiers et comptables; le droit et l'éthique; les performances et l'interopérabilité.

S'agissant des domaines d'extension, le Québec, souffrant une fois de plus d'un retard par rapport à de nombreux pays, est en cours de rattrapage. Pour ce qui est de la question du Web 2.0, le gouvernement a mené en 2011 une consultation publique, dont le rapport n'est pas encore terminé, basée sur un site Internet participatif ouvert au public. Au terme de la consultation, 113 propositions avaient été soumises par 470 utilisateurs inscrits, générant 767 commentaires et 2300 votes. L'encadré suivant dresse la liste des 20 propositions les plus populaires, et l'on reconnaîtra dans ces idées plusieurs éléments mis en avant dans cet article (ouverture des données, travail en réseau moins hiérarchique, promotion des logiciels libres, amélioration de la transparence, etc.).

8 Pour plus d'information sur le Salon du logiciel libre du Québec, voir www.s2lq.com.

9 L.R.Q., chapitre G-1.03. 


\section{LES VINGT PROPOSITIONS LES PLUS POPULAIRES DE LA CONSULTATION PUBLIQUE SUR LE WEB 2.0}

1. Vers une politique de gouvernement ouvert au Québec (127)

2. Préférer les protocoles ouverts aux plateformes propriétaires (79)

3. Une politique de données ouvertes pour le Québec (75)

4. Développement d'une nouvelle industrie de logiciels au Québec (72)

5. Tableau de bord des grands chantiers informatiques (68)

6. Exiger que les nouveaux projets logiciels soient « open source » (66)

7. Gérer les projets informatiques en mode collaboratif (54)

8. Surveillance en temps réel de l'utilisation des urgences (46)

9. Un site où les citoyens donnent leurs priorités au gouvernement (45)

10. Droit et accès des fonctionnaires à soumettre des opinions (43)

11. Avoir des animateurs de communauté (40)

12. Collaboration citoyenne - open 311 (39)

13. Autorisation de transmettre mon dossier au prochain député (38)

14. Auditer les firmes TI en cours de réalisation de contrats (35)

15. Transparence des chantiers (34)

15. Carte interactive de l'état des structures routières (34)

17. S'assurer que les outils utilisés sont en français (31)

18. Wiki interne à l'usage du gouvernement (30)

19. Bureau des services aux citoyens et des technologies innovantes (29)

20. Nouvelle technologie ou nouvelle attitude? (28)

Source : www.consultationgautrinweb2.gouv.qc.ca. Les chiffres entre parenthèses renvoient au nombre de votes au 9 février 2012.

À propos de l'idée d'ouverture des données, il s'agit - et de loin - de l'idée la plus populaire et elle recueille également de nombreux commentaires. Le gouvernement ira fort probablement dans cette direction, qui est celle suivie par de nombreux gouvernements dans le monde, y compris le gouvernement du Canada (voir la section sur les conséquences du logiciel libre). Par ailleurs, la Ville de Montréal, qui a mis en place un groupe de travail sur cette question, recommandera d'ici peu de favoriser cette politique (Deleglise, 2011). Les autres villes finiront, tôt ou tard, par suivre ce mouvement mondial. 


\section{- CONCLUSION}

Cet article a présenté le logiciel libre en répondant à cinq questions qui visaient à brosser un portrait fin et fidèle de ce mouvement de fond. Par la suite, nous avons traité de l'extension de ce mouvement à d'autres domaines que ceux du logiciel pour terminer par un survol de l'état du mouvement du libre au Québec et plus spécifiquement dans l'administration publique.

Nous avons avancé que si le gouvernement accusait un certain retard en la matière, il prenait actuellement des mesures en vue de rattraper ce retard. Malgré le fait que le logiciel libre soit robuste et qu'il ait réalisé de réels progrès en termes d'interface utilisateur, rendant aujourd'hui son usage très convivial pour un utilisateur non informaticien, on perçoit encore beaucoup de résistance au sein de l'administration. Par ailleurs, le logiciel libre s'impose dans les autres plateformes, aujourd'hui en pleine croissance (mobile, plateforme Web, infonuagique, etc.).

Il reste à déterminer si le gouvernement ira jusqu'au bout de cette transformation entamée avec la nouvelle loi, en propageant le logiciel libre au cœur de son infrastructure informationnelle - ce qui permettrait d'épargner des dizaines, voire des centaines, de millions de dollars - et en modifiant son modèle d'organisation du travail pour l'adapter à ces nouvelles pratiques. Cette transformation nécessiterait un changement majeur - ne touchant pas uniquement le secteur du logiciel - et supposerait de replacer l'humain au cœur de la grande machine gouvernementale.

Si ces nouveaux modèles soulèvent de grands défis, par exemple la protection de la vie privée posée par la recherche de plus grande transparence, il convient dès maintenant de prendre ces questions en considération, car elles offrent également de grands avantages. Dans tous les cas, nous pensons que cette révolution finira par aboutir, qu'elle soit choisie ou subie. Autant prendre les devants.

\section{BIBLIOGRAPHIE}

Apple (2011). Useful Mac OS X Terms: What is Darwin?, http://support.apple.com/kb/ TA25634 (page consultée le 27 septembre 2011).

Association francophone des utilisateurs de logiciels libres (2011). Liste des GUL francophones, http://aful.org/gul/liste (page consultée le 27 septembre 2011).

Auray, N. et M. Vicente (2006). Le logiciel libre en tant que modèle d'innovation sociotechnique: pratiques de développement et de coopération dans les communautés, www.cmo.uqam.ca/ colloques/acfas2006 (page consultée le 27 septembre 2011).

Beraud, P. et F. Cormerais (2011). "Économie de la contribution et innovation sociétale ", Innovations, $n^{\circ}$ 34, p. 163-183.

Blum, G. (2008). " Une informatique humainement acceptable est-elle possible? De l'aliénation à l'émancipation par le logiciel libre ", Nouvelles pratiques sociales, vol. $21, \mathrm{n}^{\circ} 1$, p. $52-67$.

Blum, G. et M. Ebrahimi (2007a). Activateurs de création de connaissances dans la communauté du logiciel libre Ubuntu, Actes du colloque Logiciels libres : défis et opportunités, $12^{\mathrm{e}}$ Conférence de l'Association information et management, Lausanne, 18-19 juin. 
Blum, G. et M. Ebrahimi (2007b). Impact d'une culture libre sur les modes d'organisation dans une communauté virtuelle : une étude ethnographique de la communauté du logiciel libre Ubuntu, Actes du colloque Les sciences de la gestion et la question de la liberté, Montréal, 9-10 juin.

Couture, S. et autres (2010). Un portrait de l'engagement pour les logiciels libres au Québec, www.cirst.uqam.ca/Portals/0/docs/note_rech/2010_03.pdf (page consultée le 27 septembre 2011).

Davenport, T. H. et J. C. Beck (2001). The Attention Economy: Understanding the New Currency of Business, Boston, Harvard Business Press.

Deharo, G. et A. Dimitrova (2011). « Mondialisation et technologies de communication : quelle gestion des risques par les organisations? ", Les cahiers de recherche du Centre interdisciplinaire de recherche sur le commerce extérieur et l'économie, vol. 14, p. 319-370.

Deleglise, F. (2011). "Place aux données numériques libres ", Le Devoir, 14 septembre.

Demazière, D., F. Horn et M. Zune (2006). La contribution aux logiciels libre : engagements personnels et mobilisation organisée, Communication présentée au congrès Le logiciel libre en tant que modèle d'innovation sociotechnique : pratiques de développement et de coopération dans les communautés, Montréal, 16 mai.

Di Cosmo, R. (2002). Logiciel libre pour l'administration publique : une nécessité, Bruxelles.

Elliott, M. S. (2003). The Virtual Organizational Culture of a Free Software Development Community, Communication présentée au 25th International Conference on Software Engineering, Portland, 3 mai.

Ellul, J. (1990). La technique ou l'enjeu du siècle, Paris, Économica.

Foray, D. et J.-B. Zimmermann (2001). " L'économie du logiciel libre : organisation coopérative et incitation a l'innovation ", Revue économique, vol. 52, n 5, p. 77-93.

Free Software Foundation (2004). Qu'est-ce qu'un "Logiciel Libre »?, www.gnu.org/ philosophy/free-sw.fr.html (page consultée le 27 septembre 2011).

Himanen, P. (2001). L'éthique hacker et l'esprit de l'ère de l'information, Paris, Exils.

Jullien, N. et J.-B. Zimmermann (2006). « Peut-on envisager une écologie du logiciel libre favorable aux nuls? ", Terminal, n 97-98, p. 33-47.

Ludlow, R. (2008). " Racism Ruled, Jury Finds », The Columbus Dispatch, 11 juillet, www. dispatch.com/content/stories/local/2008/07/11/Coal_Run.ART_ART_07-11-08_ A1_SPANNJH.html (page consultée le 27 septembre 2011).

Netcraft (2011). "July 2011 Web Server Survey ", Netcraft, http://news.netcraft.com/ archives/2011/07/08/july-2011-web-server-survey.html (page consultée le 27 septembre 2011).

Open Source Initiative (2007). The Open Source Definition, www.opensource.org/osd.html (page consultée le 9 février 2012).

Ordoneau, P. (2011). Passager clandestin, www.lecercle.lesechos.fr/abecedaire/p/ 221132720/passager-clandestin (page consultée le 27 septembre 2011).

Prévot, F. (2007). " Coopétition et management des compétences ", Revue française de gestion, $\mathrm{n}^{\circ} 176$, p. 183-202.

Raymond, E. S. (1999). À la conquête de la noosphère, www.linux-france.org/article/these/ noosphere/ (page consultée le 27 septembre 2011). 
Regards Citoyens (2011). Numérisons les lobbyistes : un exemple d'Open Data crowdsourcée et visualisée librement, Communication présentée aux Rencontres mondiales du logiciel libre, Strasbourg, 9-14 juillet.

Solé, A. (2000). Créateurs de mondes : nos possibles, nos impossibles, Paris, Éditions du Rocher.

Sourceforge (2011). About, www.sourceforge.net/about (page consultée le 27 septembre 2011).

Stewart, K. et S. Gosain (2006). " The Impact of Ideology on Effectiveness in Open Source Software Development Teams », MIS Quarterly, vol. 30, n² 2, p. 291-314.

Stewart, K. et S. Gosain (2001). An Exploratory Study of Ideology and Trust in Open Source Development Groups, Communication présentée à l'International Conference on Information Systems.

Von Hippel, E. (2005). " Open Source Software Projects as User Innovation Networks », dans J. Feller et autres (dir.), Perspectives of Free and Open Source Software, Cambridge, MIT Press, p. 267-278.

Weber, S. (2004). The Success of Open Source, Cambridge, Harvard University Press.

Wikipédia (2011). Wikipédia : Droit d'auteur, www.fr.wikipedia.org/wiki/Wikip \%C3 \%A9 dia:Droit_d'auteur (page consultée le 27 septembre 2011).

Williams, S., R. Stallman et C. Masutti (2010). Richard Stallman et la révolution du logiciel libre : une biographie autorisée, Paris, Eyrolles. 


\section{ANNEXE I: LES GROUPES SOCIAUX ET LES ACTIVITÉS PERMANENTES}

- L'apathie c'est plate (passer par l'art et la technologie pour sensibiliser les jeunes sur la démocratie)

-AQUOPS : Association québécoise des utilisateurs de l'ordinateur au primaire et au secondaire

-Ateliers du libre et ateliers populaires du libre

- Bande passante

- CAPE : Centre d'activités populaires et éducatives

- CASI : Laboratoire de conception et analyse de systèmes informatiques de l'École Polytechnique de Montréal

- Centre social autogéré

- Civic Access

- cliquezpourmoi.qc.ca

- Coco : English capacity development for anglo non profits in Quebec

- Collim : Comité pour le logiciel libre à Maisonneuve

- Communautique

- CS Kamouraska-Rivière-du-Loup

- Elgg Québec

-FACIL : Pour I'appropriation collective de I'informatique libre

- Fondation pour une bibliothèque globale Programme : Observatoire des SIGB (Systèmes intégrés de gestion de bibliothèques) libres

- Free Software Foundation

-Gentoo-Québec.org

- GUL Québec : Groupe d'utilisateurs du libre

- GULSE : Groupe des utilisateurs Linux de Saint-Eustache

- Gulum : Groupe d'utilisateurs Linux de I'Université de Montréal

- GULLAR : Groupe d'utilisateurs de logiciels libres à Rosemont

Source : Couture et autres, 2010.
- GULUS : Groupe d'utilisateurs Linux de I'Université de Sherbrooke

- Île sans fil

- Individu - Statistique Canada

- InukTIC

- Koumbit

- LabCMO : Laboratoire de communication médiatisé par ordinateur

- lignedutemps.qc.ca

- Limoilux

- LinuQ : Groupe de volontaires Linux à Québec

- Linux meetup

- Linux-Québec

- Liste des logiciels libres du gouvernement du Québec

- Oui-Yes Informatique

-PHP Québec

- Promotion de l'utilisation de la géomatique dans le développement de mouvements de démocratie participative

- Promotion de I'utilisation des logiciels libres dans le domaine géospatial

- QuébecOS

- Savoir Faire Linux

- Société GRICS

- SQIL : Semaine québecoise de l'informatique libre

- tribunephilosophique.org

- Ubuntu

- Ubuntu-qc

- Université du Québec à Rimouski (plateforme Claroline)

- Université du Québec en AbitibiTémiscamingue

-UQBM : Un Québec branché sur le monde

- www.culturelibre.ca

-Xo-Quebec

-Zone libre en éducation 